\title{
SIMULATIONS OF SPACE VLBI
}

\author{
DAVID W. MURPHY \\ Jet Propulsion Laboratory, MS 238-700, 4800 Oak Grove Drive, Pasadena, \\ Ca 91109, USA
}

\begin{abstract}
The use of simulations to examine various trade-offs and estimating the scientific return from the space VLBI missions RADIOASTRON and VSOP is discussed. The impact of the on-board spacecraft constraints is determined.
\end{abstract}

\section{INTRODUCTION}

In the mid 1990s we will be in the fortunate position that two space VLBI missions RADIOASTRON (USSR) and VSOP (Japan) will be operational. RADIOASTRON will be in a 24 hour orbit with an apogee height of about $70,000 \mathrm{~km}$ whereas VSOP will be in an approximately 6 hour orbit with an apogee height of $20,000 \mathrm{~km}$. VSOP will be primarily an imaging mission whereas RADIOASTRON will be primarily a high brightness temperature survey mission. Simulations are the only mechanism by which we can examine various engineering and operational trade-offs that have to.be made and to determine the scientific return from these missions. Various simulation programs exist to study these missions in the USSR, Canada, Japan and the United States and the work we describe uses our own adaptation of the Caltech VLBI package program FAKE.

\section{SPACECRAFT CONSTRAINTS}

It has become apparent in the last year that on-board spacecraft constraints seriously limit the ability of the spacecraft to undertake VLBI observations and much work has been devoted to discovering the impact of these constraints and how they may be reduced by simple redesigns of the spacecraft. For example it was proposed (Murphy, Preston, Hirabayashi and Kobayashi 1990) that the communication between VSOP and the ground tracking stations could be greatly improved by the addition of a second telemetry antenna. This however has proved impractical given the tight mass and power budgets but the single telemetry antenna may now be placed on a $70 \mathrm{~cm}$ boom compared to the 20 $\mathrm{cm}$ boom in the original design in order to improve communication geometries. For RADIOASTRON the simulation work has shown that one of the cooling constraints is so severe that a spacecraft redesign is being actively looked into at present. 


\section{$\underline{\text { ALL SKY PLOTS }}$}

Both RADIOASTRON and VSOP and have on-board spacecraft constraints for such subsystems as the solar panels, the star sensors, the telemetry antenna, the passive and active coolers and the receivers. In Figures 1 and 2 we show a set of equal area all sky projections which illustrate the percentage of the time the on-board spacecraft constraints are met as a function of epoch and celestial coordinates i.e. the percentage of the time data can be taken. For the VSOP simulation we assumed a launch date of 30 January 1995 with an orbit having an initial argument of perigee of $90^{\circ}$, an RA of the ascending node of $0^{\circ}$, an inclination of $46^{\circ}$, and apogee and perigee heights of $20,000 \mathrm{~km}$ and $1,000 \mathrm{~km}$ respectively. The first two parameters in this list precess at a rate of $180^{\circ}$ per year which leads to a very strong epoch dependence caused by the fact that tracking stations are preferentially located in the Northern Hemisphere. For the RADIOASTRON simulation the launch date is chosen to be 9 May 1993 with an orbit having an argument of perigee of $225^{\circ}$, an RA of the ascending node of $0^{\circ}$, an inclination of $65^{\circ}$, and apogee and perigee heights of $70,000 \mathrm{~km}$ and $1,500 \mathrm{~km}$ respectively. With this choice of orbital parameters the perigee does not precess and the RA of the ascending node precesses only very slowly. The time evolution of the RADIOASTRON constraints is caused by the interaction of the solar constraints with the other spacecraft constraints. As can be seen, for both missions, the spacecraft constraints limit programs such as superluminal monitoring.

\section{CONCULSIONS}

The impact of the on-board spacecraft constraints for both RADIOASTRON and VSOP is substantial. The impact of these constraints needs to be understood and various studies need to be performed to see how the effects can be minimized either by spacecraft redesign, choice of orbits or by careful scheduling during the mission.

\section{ACKNOWLEDGEMENTS}

I would like to thank Bob Preston for his comments on the manuscript and to colleagues in Japan, USSR and Canada for the very fruitful intercomparison of software that has taken place during the last year. This work was carried out while DWM held a National Research Council/NASA Research Associateship at the Jet Propulsion Laboratory.

\section{REFERENCES}

Murphy, D.W., Preston, R.A., Kobayashi, H. and Hirabayashi, H. 1990, in Frontiers of VLBI, ed. H. Hirabayashi, M. Inoue and H. Kobayashi, Universal Academy Press, in press. 

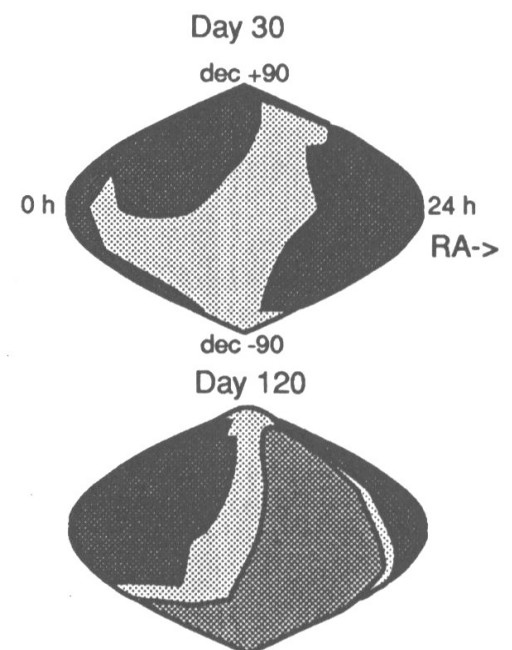

Day 210

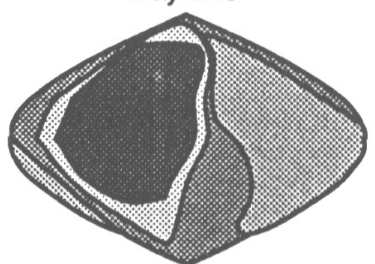

Day 300

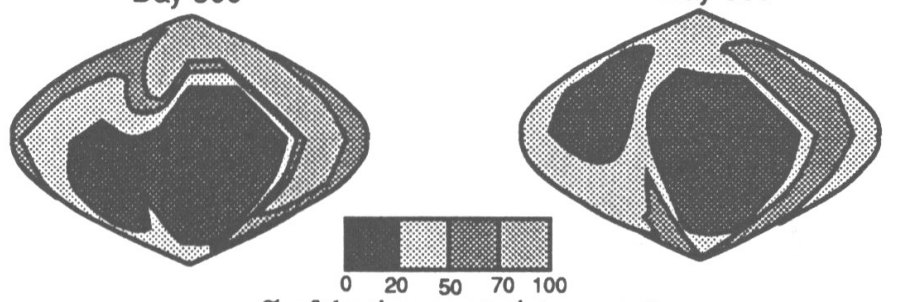

$\%$ of the time constraints are met
Day 390

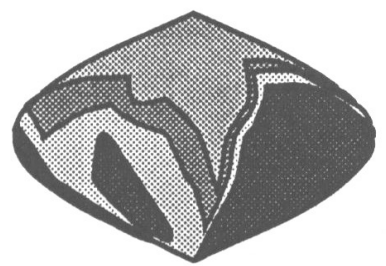

Day 480

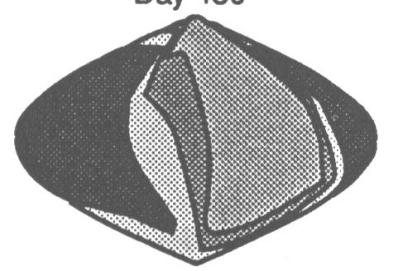

Day 570

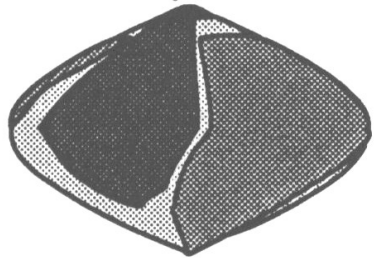

Day 660

Fig. 1 Time evolution of the VSOP constraints. 

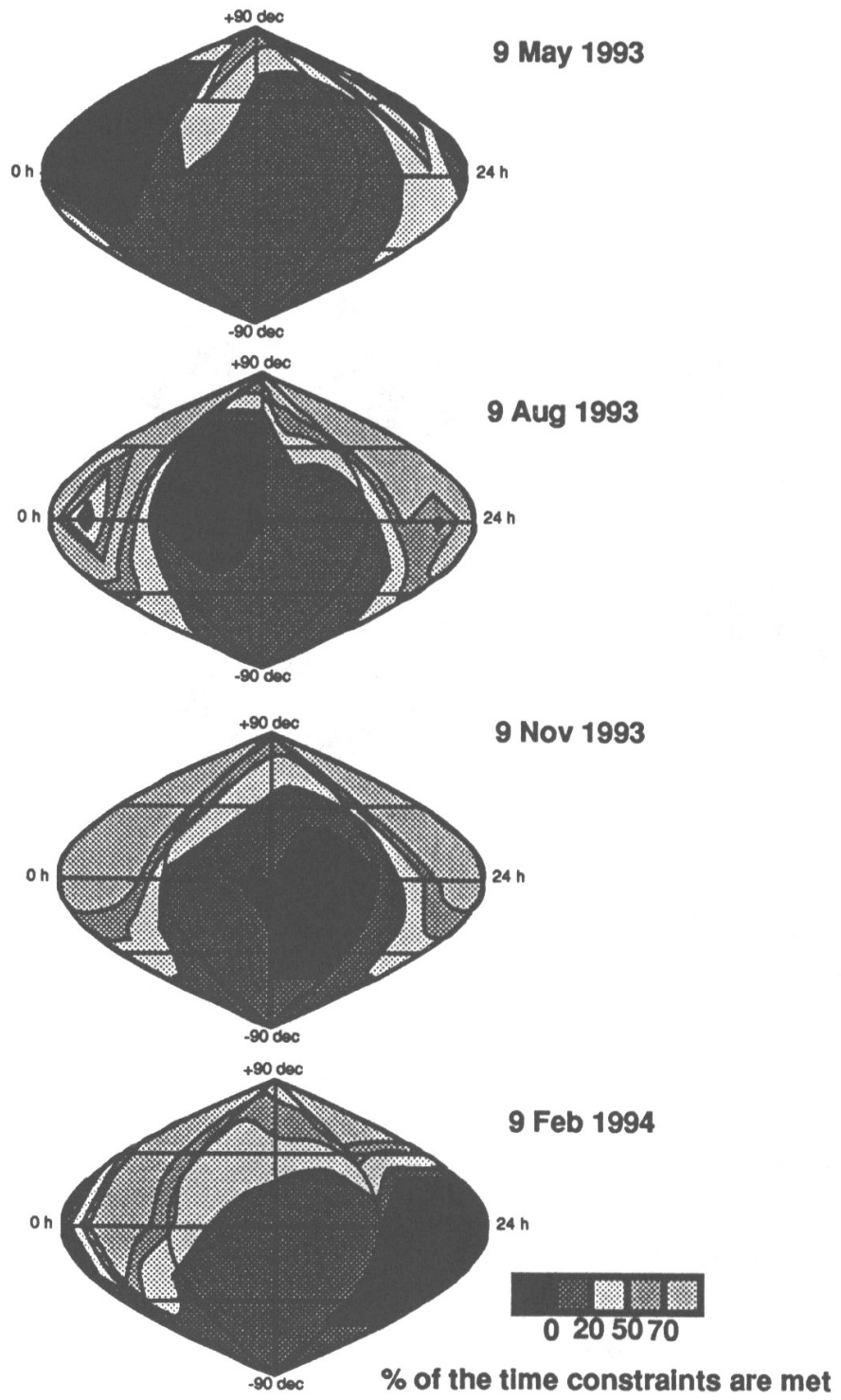

Fig. 2. Time evolution of the RADIOASTRON constraints. 
Richard Porcas: I think it would be very desirable to consider a further constraint in your simulations, namely, the limited availability of time at ground VLBI facilities. Although general commitments of time to space VLBI projects have been made, it is clear that, because of other observing programs, time will only be made available in blocks, probably of length about a week or two. How will this compromise the $u, v$ coverage, and how efficiently can the ground VLBI facility time be used? Perhaps it would be useful to simulate a 2week long observing program with 'famous' sources to examine the implications for scheduling the ground facilities.

Dave Murphy: I hope to examine the scheduling of space VLBI and how to optimize the use of ground arrays in the near future. With both VSOP and Radioastron operational concurrently, scheduling is made even more difficult. The space agencies and ground arrays need to examine what is expected of each other.

Larry D'Addario: You said that there is a shortage of tracking stations in the Southern hemisphere. Why doesn't NASA put one of its new stations in, say, Argentina, rather than California or Spain?

David Murphy: I agree that there are benefits to placing one of the northern hemisphere tracking stations, say Goldstone or Green Bank, in Argentina. These benefits must be weighed against increased cost, loss of redundancy and the increased tracking conflicts between VSOP and Radioastron. 